\title{
Un ciclo de mejora docente basado en la resolución de problemas aplicado a la asignatura de Prehistoria I
}

\author{
Álvaro Gómez Peña \\ Universidad de Sevilla \\ Departamento de Prehistoria y Arqueología \\ agomez19@us.es \\ ORCID: https://orcid.org/0000-0002-2926-5243 \\ D.O.I.: http://dx.doi.org/10.12795/JDU.2018.i01.25 \\ Pp.: 450-465
}

\section{Resumen}

En la presente publicación se detallan las características del ciclo de mejora aplicado a los cuatro primeros temas impartidos al grupo 3 de la asignatura 'Prehistoria I' dentro del Grado de Historia de la Universidad de Sevilla durante el curso académico 2018/2019. El ciclo de mejora ha consistido en la aplicación de aprendizaje por problemas aplicado a una ciencia básica de tipo histórico. En las siguientes páginas se expone el modelo ideal y se comentan los problemas, soluciones y mejoras a aplicar en próximos cursos.

Palabras clave: Prehistoria I, Historia, Docencia universitaria, Aprendizaje Basado en Problemas, Modelo TORA 


\section{Breve descripción del contexto}

El ciclo de mejora realizado como parte del proyecto de innovación docente se ha aplicado a la asignatura Prehistoria I dentro del Grado de Historia siguiendo los parámetros principales incluidos en la obra Enseñanza Universitaria. Cómo mejorarla (Porlán 2017). Se trata de una asignatura obligatoria impartida en el primer cuatrimestre del primer curso de dicho grado. El conocimiento que suele tener el alumnado sobre sus contenidos es casi nulo por dos motivos principales: por un lado, el escasísimo tratamiento de esta materia en secundaria y bachillerato, más volcado a impartir docencia de Historia Contemporánea, y por otra parte la nula formación obtenida en la propia carrera por ser una de las primeras asignaturas que cursan.

Las clases impartidas han tenido lugar en el Aula Magna de la Facultad de Geografía e Historia. Se trata de un espacio especialmente grande y escalonado, como los cines modernos, donde los estudiantes que habitualmente asisten a clase no suelen tener problemas para ocupar los asientos y conectar sus portátiles si lo necesitan. Es un aula con suficiente iluminación natural y artificial. La mesa del profesor se encuentra sobre una gran tarima, lo que facilita la comunicación visual. Dado que la clase es especialmente amplia, es necesario contar con un micrófono para que el volumen no se pierda. Suele funcionar y además bastante bien. Igualmente, hay un proyector y una buena pantalla conectados al ordenador que hay sobre la mesa del docente. También la pizarra es amplia, aunque no cuenta con luz sobre ella para que se vea mejor lo que en ella se escribe.

El horario de la clase es de 15 a 16h de lunes a jueves. Se trata de la primera sesión que los alumnos reciben por la tarde. También es la primera que imparto en el día. Se nota que tanto ellos como yo estamos frescos y normalmente ha habido una buena participación en clase. 
Este curso académico he compartido la docencia. Yo me encargo de dar los 5 primeros créditos con carga teórica y un compañero hace lo propio con el crédito restante enseñándole a los estudiantes en las clases prácticas los tipos de industria lítica para que sepan cómo identificarlas, determinar sus partes, funciones, cronologías y por último dibujarlas dentro del proceso lógico de investigación desarrollado con este tipo de piezas.

\section{Diseño previo del Ciclo de Mejora Docente}

Hace varios cursos tuve la oportunidad de impartir esta misma asignatura a estudiantes de primer curso en horario de mañana. Los contenidos de mi asignatura no corresponden con mi especialidad de investigación. Eso me generó un especial estrés porque, además de tener que enseñar sus contenidos, tuve al mismo tiempo que ir aprendiéndolos para poder impartirlos dado que el profesor que me dio clases de Prehistoria durante mi licenciatura era un auténtico desastre. Aquella situación me generó dos problemas. Por una parte, no conocía ni los límites, ni la totalidad de los contenidos de la asignatura que impartía con anterioridad a su comienzo. Por otro lado, derivado de este hecho, no podía de antemano saber cómo estructurar los contenidos ni cómo desarrollar estrategias a partir del material didáctico disponible para hacerlo.

Este curso la situación ha cambiado bastante. Es mi tercer año impartiendo la asignatura, me motivan mucho sus contenidos, los empiezo a conocer bastante bien, también su bibliografía específica asociada, así como las novedades que ocurren en esta área de estudio año tras año. Igualmente, empiezo a ganar experiencia para saber cuáles son los principales problemas y ventajas que suelen tener los alumnos y alumnas con cada tema para ir haciendo pequeñas modificaciones. Este año me siento por lo tanto más capacitado para ampliar el CMD a un número más elevado de clases. 
Las clases habituales en años anteriores han consistido en lecciones magistrales apoyándome en determinadas ocasiones en material didáctico: extractos de libros para comentar en clase y vídeos breves, principalmente. En todos los casos, el uso de estos recursos era secundario y siempre como complemento de los contenidos impartidos de modo magistral.

Frente a este tipo de clases tradicionales, mi ideal de enseñanza quiero que se base en un aprendizaje basado en la resolución de problemas para que los estudiantes vayan poco a poco cogiendo el hábito de trabajar y reflexionar en el aula como tendrán que hacerlo posteriormente a la hora de desempeñar profesiones relacionadas con la Historia.

Dentro de cada tema en sí, las sesiones comenzarían (y de hecho ya lo hago a veces con buenos resultados) llevando a los estudiantes recursos bibliográficos sobre los aspectos a tratar en esa y posteriores clases para que durante esa hora de clase quien quiera pueda consultar la obra, ver su indice, conocer la reseña del autor, su nombre, bibliografía relacionada con esa temática al final del libro, etc. Tras unos primeros minutos de presentación de la obra llevada a clase, plantearía una cuestión que tenga relación con un problema teórico, metodológico o factual del pasado o del presente con la intención de generar un primer debate entre los alumnos y alumnas.

En todos los casos, inmediatamente trataría de que se generara un debate con las ideas e hipótesis de los estudiantes y sus posibles argumentos para retractarlas o defenderlas. Mi labor en esta etapa de la sesión sería por un lado plantear nuevas preguntas menores para complejizar y hacer evolucionar el debate, y por otra parte apuntar y ordenar estructuralmente las ideas en la pizarra para realizar posteriores aclaraciones. La lección hasta este punto habría servido para plantear posibles alternativas a un esquema preestablecido sobre el asunto que tuvieran los estudiantes (si es que lo tenían). Desde mi punto de vista, la 
Universidad sirve para generar reflexiones, no para dogmatizar pseudo-objetividades y axiomas.

En una tercera fase dentro de la sesión de una hora, habría lugar para reordenar las ideas ya visibles en la pizarra y empezar a profundizar en ellas. La estructura de contenidos de esta sección se basaría en explicar las diferentes hipótesis propuestas para dar respuesta a la pregunta, analizar los argumentos en que se basan, y en los casos en que la pregunta tenga estrecha relación con los datos arqueológicos mostrar a los estudiantes cuáles son esas piezas y contextos arqueológicos en que se basan las hipótesis. Dentro de este segundo apartado sería especialmente interesante que los alumnos y alumnas tuvieran material de apoyo. Me gustaría hacer especial hincapié en el empleo de libros y artículos donde los principales autores que hayan tratado la cuestión a debate en la clase desarrollen sus ideas para que los estudiantes empiecen a ganar el hábito de consultar y contrastar la información de primera mano, conociendo autores, títulos y editoriales de referencia. La Historia es en muchas ocasiones una actividad donde principalmente se lee para extraer información, y dado que la experiencia práctica en relación con la parte arqueológica la desarrolla mi compañero, considero que mis clases son el momento perfecto para desarrollar este asunto.

Por último, al final de cada tema se pasaría un nuevo cuestionario a los estudiantes para comprobar el nivel de aprendizaje obtenido comparando el antes y el después.

\section{Mapa de contenidos de la asignatura}

El Cuadro 1 recoge ordenados estructuralmente los temas que se imparten en la asignatura de Prehistoria I. En letras blancas aparecen los que se verían afectados por el CMD. Como se puede observar, se parte en los primeros temas de aspectos más generales a temas más específicos. Desde el método científico y sus particularidades en el 
estudio de la prehistoria, pasando por las diferencias corrientes epistemológicas. El tema 3 y el tema 8 son equivalentes a los tiempos Paleolítico y Neolítico respectivamente, por lo que los temas 4-7 y 9 están estrechamente vinculados a ambos.

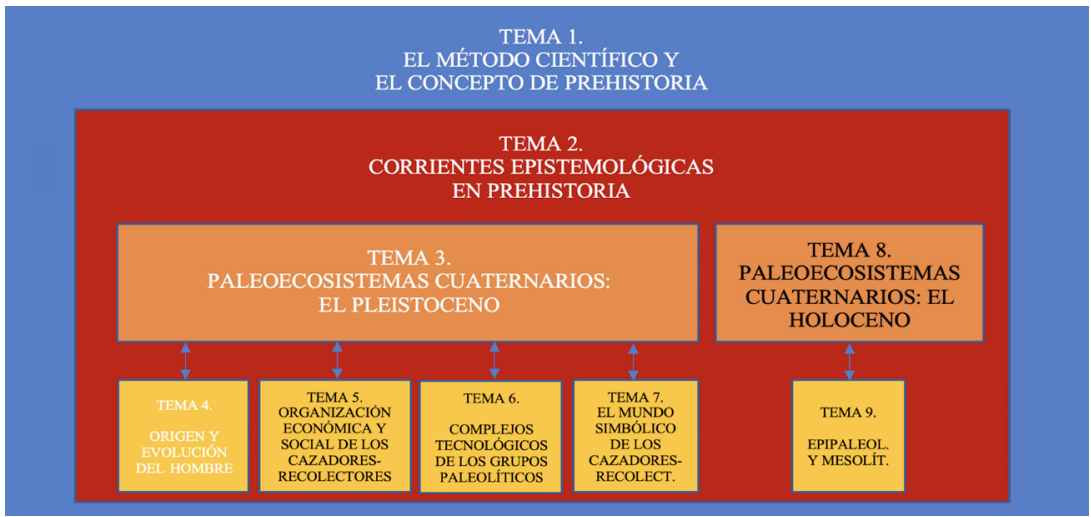

Figura 1. Cada cuadro de color indica que el marco de influencia sobre otros temas. En letras blancas aparecen los temas que se verían afectados por el CMD.

Hay temas rectores (fig. 1) que siempre van a aparecer periódicamente a lo largo del desarrollo de otros posteriores (1-2) por los contenidos filosóficos y metodológicos explicados en ellos, mientras que hay otros temas (3-9) que presentan relaciones recíprocas. En este sentido, el marco paleoambiental del Pleistoceno y del Holoceno condicionan la evolución homínida y su cultural material, mientras que la propia acción de los homínidos sobre su nicho ecológico condiciona las características de los biomas en lo que éstos viven. A esta relación mutua hacen referencia las flechas que conectan unos temas con otros.

En el desarrollo de este CMD, dado que se tocan los 4 niveles de desarrollo de la asignatura, se puede observar muy bien el efecto cascada que los primeros debates pueden llegar a tener en las discusiones y aportaciones de ideas sobre temas propiamente arqueológicos (4-7 y 9). Sería interesante observar el nivel de aprendizaje de los estudiantes en las cuestiones anteriores a cada nuevo tema 


\section{Modelo metodológico posible y secuencia de actividades programada, partiendo de los problemas seleccionados}

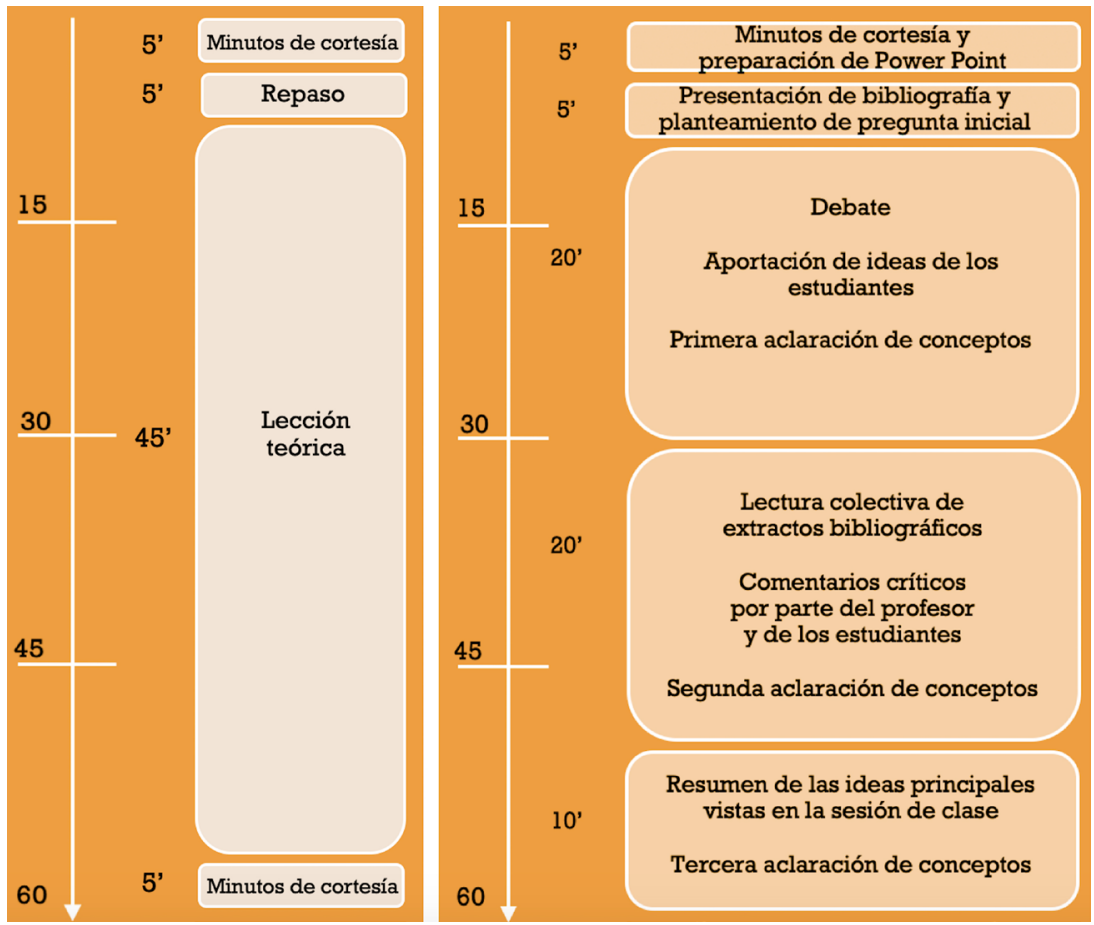

Figura 2. Estructura de clase tradicional y estructura de clase aplicando el CMD.

Frente a la clase magistral tradicional, compuesta por $5^{\prime}$ de cortesía y preparación de Power Point, 5' de repaso y $45^{\prime}$ de clase magistral con la inserción de textos y vídeos cuando se fuese llegando durante las sesiones a determinados apartados, la nueva propuesta basada en resolución de problemas (problemas conceptuales y metodológicos en su aplicación al estudio teórico de la Prehistoria) es la aquí recogida (fig. 2). 
Cuestionario inicial-final para hacer un seguimiento de la evolución de los estudiantes en relación a los problemas clave

Se han realizado 4 cuestionarios iniciales y finales, uno por cada tema visto en clase dentro del CMD. En todos los casos, las preguntas iniciales y finales han sido las mismas, excepto la última pregunta del cuestionario final. He de reconocer que he tenido numerosas dudas a la hora de sintetizar las preguntas que quería proponerle a los estudiantes, no por no tener claros los conceptos que quería que aprendiesen, sino para reflejar de modo sencillo y directo algunos de los problemas fundamentales que quería que contestaran sin darles muchas pistas en preguntas posteriores. Finalmente, los temas y las preguntas planteadas han sido las siguientes:

Tabla 1. Preguntas por tema realizadas en la evaluación previa y posterior.

\begin{tabular}{|c|c|c|c|c|c|}
\hline \multicolumn{6}{|c|}{ TEMA 1. El método cientifico y el concepto de Prehistoria } \\
\hline $\begin{array}{l}\text { ¿Qué es la } \\
\text { Prehistoria? }\end{array}$ & $\begin{array}{l}\text { ¿Cómo se obtienen los } \\
\text { datos en Prehistoria? }\end{array}$ & $\begin{array}{l}\text { ¿La Prehistoria } \\
\text { forma parte de la } \\
\text { Historia? }\end{array}$ & $\begin{array}{l}\text { ¿La Historia es una } \\
\text { disciplina cientifica? }\end{array}$ & $\begin{array}{c}\text { ¿Cómo se } \\
\text { hace ciencia? }\end{array}$ & $\begin{array}{c}\text { ¿Es posible hacer } \\
\text { ciencia desde la } \\
\text { disciplina prehistórica? } \\
\text { ¿Por qué? }\end{array}$ \\
\hline \multicolumn{6}{|c|}{ TEMA 2. Corrientes epistemológicas en Prehistoria } \\
\hline $\begin{array}{l}\text { ¿Cuándo } \\
\text { surge la } \\
\text { disciplina } \\
\text { prehistórica? }\end{array}$ & $\begin{array}{c}\text { ¿Cómo surge la } \\
\text { disciplina } \\
\text { prehistórica? }\end{array}$ & $\begin{array}{l}\text { ¿Qué es la } \\
\text { epistemología? }\end{array}$ & $\begin{array}{c}\text { ¿Qué corrientes } \\
\text { teóricas y/o autores } \\
\text { conoces para } \\
\text { interpretar la Prehist.? }\end{array}$ & $\begin{array}{c}\text { ¿Por qué } \\
\text { cambian las } \\
\text { teorías en } \\
\text { Prehistoria? }\end{array}$ & $\begin{array}{l}\text { ¿Tiene sentido la } \\
\text { filosofia de la } \\
\text { Prehistoria? ¿Por qué? }\end{array}$ \\
\hline \multicolumn{6}{|c|}{ TEMA 3. Paleoecosistemas cuaternarios: El Pleistoceno } \\
\hline $\begin{array}{l}\text { ¿Qué es el } \\
\text { Pleistoceno? }\end{array}$ & $\begin{array}{l}\text { ¿Qué caracteriza el } \\
\text { Pleistoceno? }\end{array}$ & $\begin{array}{l}\text { ¿Existe el cambio } \\
\text { climático? }\end{array}$ & $\begin{array}{l}\text { ¿Cómo definirías el } \\
\text { cambio climático? }\end{array}$ & $\begin{array}{c}\text { ¿Desde } \\
\text { cuándo existe } \\
\text { cambio } \\
\text { climático? }\end{array}$ & $\begin{array}{l}\text { ¿La fauna y la flora ha } \\
\text { sido siempre la misma } \\
\text { en las mismas regiones } \\
\text { del planeta? ¿Por qué? }\end{array}$ \\
\hline \multicolumn{6}{|c|}{ TEMA 4. Origen y evolución del hombre } \\
\hline $\begin{array}{l}\text { ¿Cuándo y } \\
\text { dónde } \\
\text { surgió el ser } \\
\text { humano? }\end{array}$ & $\begin{array}{l}\text { ¿Sabrías describir a un } \\
\text { Australopithecus/y } \\
\text { nombrarme algunas } \\
\text { especies de su género? }\end{array}$ & $\begin{array}{l}\text { ¿Sabrías describir a } \\
\text { un Paranthropus y } \\
\text { nombrarme algunas } \\
\text { especies de su } \\
\text { género? }\end{array}$ & $\begin{array}{l}\text { ¿Qué características } \\
\text { anatómicas y } \\
\text { conductuales forman } \\
\text { parte del género } \\
\text { Homo? }\end{array}$ & $\begin{array}{l}\text { ¿Cómo y por } \\
\text { qué surgen } \\
\text { las especies? }\end{array}$ & $\begin{array}{l}\text { ¿Los humanos somos } \\
\text { diferentes al resto de } \\
\text { especies? ¿Por qué? }\end{array}$ \\
\hline
\end{tabular}

En líneas generales, y teniendo en cuenta que no todos los estudiantes que realizaron las preguntas iniciales respondieron a las finales por faltar a alguno de los dos días, de los 54 alumnos matriculados han respondido al menos 6 de los 8 cuestionarios un total de 40 estudiantes, lo que ha hecho posible observar la evolución en los conocimientos adquiridos antes y después de cada tema de un $75 \%$ de la clase, cifra suficientemente representativa; sobre 
algunas particularidades de la evaluación hablaré a continuación en el apartado 3.2.

\section{Aplicación del CMD}

\section{Relato resumido de las sesiones}

La aplicación del CMD se ha llevado a cabo entre los días 25 de septiembre y 15 de noviembre en un total de 30 horas lectivas.

La propuesta de CMD contemplaba la realización de sesiones monotemáticas de 60 minutos en las cuales tratar diferentes subtemas englobados dentro del tema principal que se estuviera dando en cada momento. En este punto, no ha habido especiales problemas y en la mayoría de los casos se ha podido cumplir el programa establecido de antemano. De hecho, el tema 4 se ha terminado justo el 15 de noviembre.

Una de las mayores dificultades la he encontrado a la hora de respetar los tiempos previstos para cada fase en la que he pretendido dividir la clase. En algunas ocasiones no me ha sido sencillo controlar el tiempo de debate o el de lectura con comentarios críticos. En unos casos porque los debates se han alargado más del tiempo previsto (a ello ha contribuido la presencia de alumnos del aula de la experiencia que ha facilitado dinamizar las participaciones) y en otros porque los estudiantes tenían más claras de lo que pensaba algunas cuestiones (lo que ha permitido profundizar más en algunas partes del temario y enriquecer con más datos e hipótesis los asuntos a tratar); en varias ocasiones me ha preocupado andar mirando a menudo el tiempo restante de clase, sobre todo en momentos en los que la clase estaba siendo más dinámica e interesante. 
En algunas sesiones, especialmente en aquellas que han afectado a los contenidos del tema 4, la lectura de textos se ha sustituido por el visionado de fragmentos del documental sobre evolución hominina titulado La odisea de la especie en los que autores como Juan Luis Arsuaga o Yves Coppens realizaban pequeñas intervenciones sobre aspectos observados en la teatralización de la vida de los diferentes primates bípedos. Este documental en años anteriores ha tenido muy buena acogida por los estudiantes porque les permite comprender de un modo más cercano algunos datos y rasgos evolutivos fundamentales debatidos en clase.

Por otra parte, en algunas ocasiones he tenido que variar el orden del CMD y realizar breves explicaciones de sintesis justo al acabar el debate para profundizar en algunas ideas, especialmente vinculadas con los conceptos de selección natural y estrategias reproductivas de la fauna pleistocénica antes de leer algunos textos. Este asunto tengo que repensarlo en cursos venideros porque en los casos en los que he invertido el orden de los dos grandes bloques del final de la sesión ha sido porque los alumnos estaban interesados tras el debate en profundizar más en algunos temas que iban saliendo a colación en el debate, asunto que me ha gratificado especialmente porque he visto que el interés de los estudiantes iba más allá de la materia que tenía prevista.

Aunque no tengo datos comparativos entre este curso y cursos anteriores, he observado un impacto significativo con la implementación del CMD en los estudiantes a lo largo de las horas de clase porque han estado más participativos. Tengo la impresión de que el momento de debate les ha permitido promover su espíritu crítico y aclarar con la ayuda de otros compañeros algunas ideas previas, por pocas que fueran, sobre la materia a tratar. De hecho, este año ha habido más alumnos interesados en realizar trabajos voluntarios que en cursos anteriores. 
Evaluación del aprendizaje de los estudiantes, comparando los conocimientos iniciales y finales

El proceso de evaluación de los conocimiento iniciales y finales se ha realizado sobre un total de 54 estudiante matriculados. Los resultados pre y post-tema revelan claras mejorías al menos en el $80 \%$ de los estudiantes, especialmente en aquellos que asisten regularmente a clase. No obstante, los resultados no son concluyentes. Solamente he podido aplicar un ciclo de mejora similar en esta asignatura durante el curso 2013/2014 y su aplicación se realizó sobre el tema 7 . No es posible por tanto comparar los resultados de ambos cursos. Por otra parte, desde dicho curso hasta el curso 2017/2018 no volví a impartir la materia de Prehistoria I. En ninguno de estos cursos realicé evaluaciones iniciales y finales dentro de un sistema de enseñanza principalmente magistral. En este sentido, los resultados obtenidos durante este curso académico suponen el inicio de un conjunto de evaluaciones pre y posttema que podrán empezar a ser comparados en cursos posteriores. Será sin lugar a dudas una de las iniciativas que continúe implementando en futuros cursos al continuar muy probablemente impartiendo esta asignatura.

Jornadas de Formación e Innovación Docente del Profesorado | № 1 (2018) Esta obra se distribuye con la licencia Creative Commons 


\section{TEMA 1}

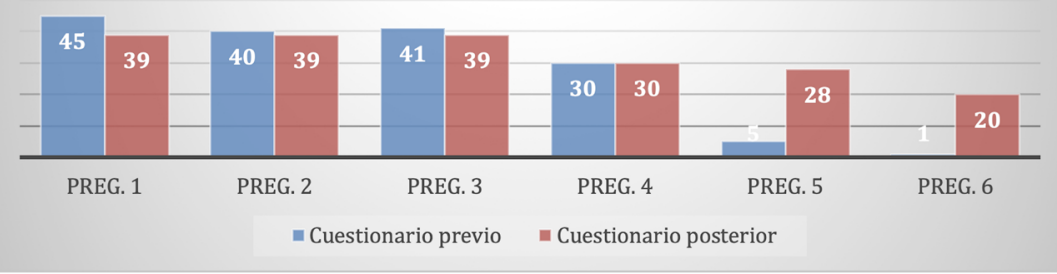

\section{TEMA 2}

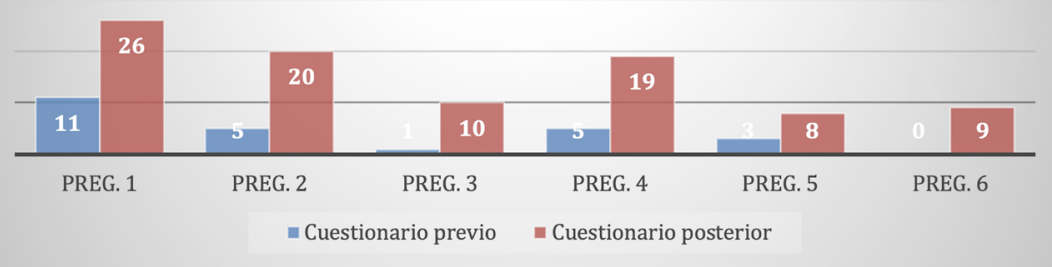

\section{TEMA 3}

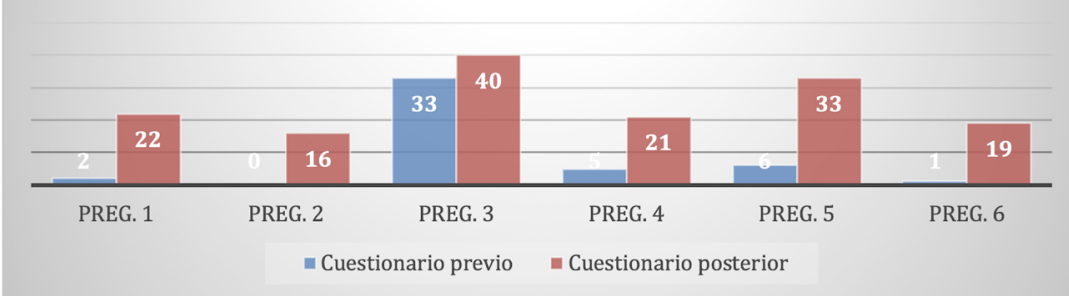

\section{TEMA 4}

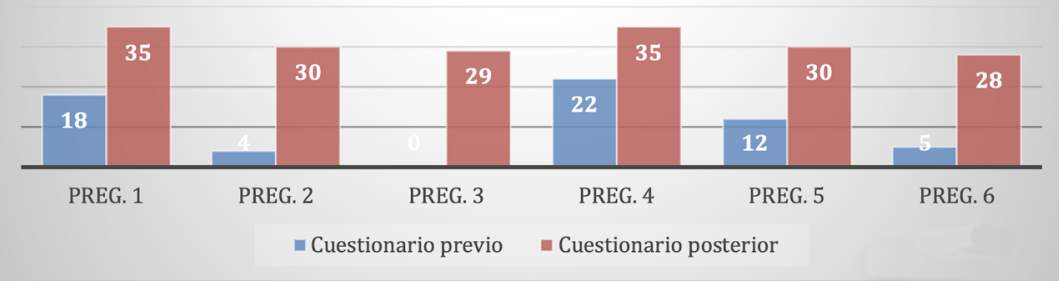

Figura 3. Gráfica con las respuestas correctas de los estudiantes en los cuestionarios previos y posteriores de los temas sobre los que se ha aplicado el CMD.

Jornadas de Formación e Innovación Docente del Profesorado I № 1 (2018) (c) (i) $(-)$ Esta obra se distribuye con la licencia Creative Commons Reconocimiento-NoComercial-SinObraDerivada Internacional (CC BY-NC-ND 4.0.) 


\section{Evaluación del CMD puesto en práctica}

\section{Cuestiones a mantener y cambios a introducir para un futuro Ciclo de Mejora más amplio}

La estructura generada este año para elaborar el CMD, consistente en una pregunta de inicio, un debate, una aclaración de conceptos, lecturas críticas con preguntas y respuestas, segunda aclaración de conceptos y resumen final con tercera aclaración de conceptos como cierre de clase ha dado muy buenos resultados. En líneas generales he visto más asistencia a clase y una mayor participación que en cursos y asignaturas impartidas con anterioridad. Volveré a repetir este modelo.

El uso de un cuestionario previo y final lo había implementado en años anteriores y me parece una manera maravillosa de conocer el nivel de los estudiantes antes de trabajar los contenidos del temario. De este modo puedo saber no sólo cuál es dicho nivel, sino los errores conceptuales que tienen de antemano, lo que me permite saber cómo afrontar las explicaciones y las preguntas de los diferentes debates.

La aplicación del modelo TORA, comentado en el epígrafe 4.2. me parece un modelo muy bueno para generar interés, orden y relevancia en los estudiantes. Lo utilicé en el anterior ciclo de mejora que impartí durante el curso 2014/2015 con muy buenos resultados. Los estudiantes me decían que podían coger apuntes con mayor facilidad, que las clases no se saturaban de contenidos diversos y que si se faltaba a alguna clase, era sencillo seguir el hilo argumental de cada sesión por separado dado que cada hora de clase era el marco para desarrollar un subtema con inicio y final.

Con respecto a los cambios y mejoras a implementar, reconozco que tengo que saber manejar mejor los tiempos para no alargar demasiado la fase del debate o la de 
las lecturas críticas, sobre todo teniendo en cuenta que cada sesión de una hora es un microcosmos conceptual cerrado y no quiero dejar para posteriores clases algunas ideas sueltas que no haya dado tiempo a ver en la sesión anterior.

Es posible que implemente el uso de alguna herramienta informática como Kahoot! para realizar las evaluaciones y tener recogidos los datos en la plataforma online para manejarlos más fácilmente.

\section{Principios didácticos argumentados que han guiado la experiencia y que deben permanecer en el futuro}

Entre los principios didácticos que han guiado la experiencia docente se encuentra como punto centra el aprendizaje basado en problemas. Ha sido especialmente productivo el cambio de modelo y he notado una diferencia notable entre los temas impartidos de este modo y los temas impartidos a la manera tradicional, especialmente en la participación de los estudiantes. En este sentido, el uso de preguntas constantes como eje vertebrador de la docencia ha gustado a varios alumnos que me lo han planteado abiertamente al final de las clases, especialmente los estudiantes de más edad, que suelen tener menos problemas para expresarte sus ideas.

Por otro lado, el empleo de cuestionarios previamente y con posterioridad a la impartición de la materia es un buen termómetro para conocer el nivel de los estudiantes, la impronta de lo aprendido y los problemas que presenta su aprendizaje cuando dan respuestas erróneas. Desde mi punto de vista esta última es el mayor valor que tienen los cuestionarios y las escaleras de aprendizaje.

Más allá de los cuestionarios para evaluar los conocimientos de los estudiantes en el CMD o del modelo de aprendizaje por problemas (conceptuales, propios de la ciencia básica histórica) que planteo a los discentes, en 
paralelo hay un modelo de trabajo que quiero seguir desarrollando en las aulas y que hunde sus raíces en la propuesta TORA realizada por S. Ham para realizar interpretación del patrimonio (Ham 1992). Bajo estas siglas se engloban explicaciones temáticas, organizadas, relevantes y atractivas. En este tipo de explicaciones hay que llamar la atención sobre la necesidad de encabezar el discurso con un tema de interés para la audiencia (Thorndyke 1977). Igualmente es importante tener presente el número de ideas a tratar en cada clase para no saturar a los estudiantes. Durante un tiempo pareció útil recurrir al llamado "Principio del $7+/-2$ ". Este principio fue desarrollado por George Miller (1956) y llama la atención sobre el número de ideas nuevas a las que de promedio los adultos jóvenes son capaces de memorizar a corto plazo la primera vez que las escuchan. En consecuencia, si estamos interesados en que todo el mundo capte nuestro mensaje al cien por cien el número de ideas principales debe ser siempre menor o igual que 5. Sin embargo, estudios posteriores (Cowan 2001) recomiendan trabajar con un nuevo concepto, el del "Número mágico 4", que limita las ideas principales a este valor para el mismo espectro de la sociedad. 


\section{Referencias bibliográficas}

Cowan, N. (2001). The magical number 4 in short-term memory: A reconsideration of

mental storage capacity, Behavioral and Brain Sciences, 24 (1), 87-114.

Ham, S. (1992). Environmental interpretation: A practical guide for people with big ideas and small budgets. Golden: North American Press.

Miller, G. A. (1956). The magical number seven, plus or minus two: Some limits on our

capacity for processing information, Psychological Review, $63(2), 81-97$.

Morales, J. \& Ham, S. (2008). ¿A qué interpretación nos referimos?, Boletín de Interpretación, 19, 4-7.

Porlán Ariza, R. (coord.) (2017). Enseñanza Universitaria. Cómo mejorarla. Madrid: Ediciones Morata.

Thorndyke, P. W. (1977). Cognitive Structures in Comprehension and Memory of Narrative Discurse, Cognitive Psychology, 9 (1), 77-110.

Jornadas de Formación e Innovación Docente del Profesorado | № 1 (2018) Esta obra se distribuye con la licencia Creative Commons 